\title{
El servicio delivery, como tendencia microempresarial, ante la emergencia sanitaria por el COVID-19, en la ciudad de Cuenca
}

The delivery service, as a micro-business trend, in the face of the health emergency caused by COVID-19, in the city of Cuenca

1 Edison Becerra Molina https://orcid.org/0000-0002-6397-9493

Universidad Católica de Cuenca, Carrera de Contabilidad y Auditoría, provincia Azuay

jbecerram@ucacue.edu.ec

2 Oscar Calle Masache https://orcid.org/0000-0002-1605-9555 Universidad Católica de Cuenca, Carrera de Contabilidad y auditoría, provincia Azuay ocalle@ucacue.edu.ec

3 Pedro Astudillo Arias https://orcid.org/0000-0003-3088-8322 Universidad Católica de Cuenca, Carrera de Contabilidad y auditoría, provincia Azuay pastudillo@ucacue.edu.ec

$4 \quad$ Remigio Ojeda Orellana iD https://orcid.org/0000-0003-2691-0638 Universidad de Cuenca, Ciencias Económicas y Administrativas, provincia Azuay remigio.ojeda@ucuenca.edu.ec

Artículo de Investigación Científica y Tecnológica Enviado: 10/12/2021 Revisado: 27/12/2021

Aceptado: 22/01/2022

Publicado:25/02/2022

DOI: https://doi.org/10.33262/visionariodigital.v6i1.2076

Cítese: $\quad$ Becerra Molina, E., Calle Masache, O., Astudillo Arias, P., \& Ojeda Orellana, R. (2022). El servicio delivery, como tendencia microempresarial, ante la emergencia sanitaria por el COVID-19, en la ciudad de Cuenca. Visionario Digital, 6(1), 89-112. https://doi.org/10.33262/visionariodigital.v6i1.2076

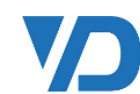

Ciencia Digital

VISIONARIO DIGITAL, es una Revista Científica, Trimestral, que se publicará en soporte electrónico tiene como misión contribuir a la formación de profesionales competentes con visión humanística y crítica que sean capaces de exponer sus resultados investigativos y científicos en la misma medida que se promueva mediante su intervención cambios positivos en la sociedad. https://visionariodigital.org

La revista es editada por la Editorial Ciencia Digital (Editorial de prestigio registrada en la Cámara Ecuatoriana de Libro con No de Afiliación 663) www.celibro.org.ec

Esta revista está protegida bajo una licencia Creative Commons Attribution Non Commercial No Derivatives 4.0 International. Copia de la licencia: http://creativecommons.org/licenses/by-nc-nd/4.0/. 


Palabras
claves:
COVID-19,
servicio
delivery,
consumidores,
aplicaciones
móviles.

\section{Resumen}

Introducción. Durante la pandemia del COVID-19 surgió la necesidad de crear aplicaciones móviles para buscar la manera de llevar productos a los clientes que no desean exponerse, esta situación origino el nacimiento de nuevos emprendimientos que perduren aun cuando la pandemia sea controlada, es ahí donde nacen empresas que prestan el servicio de entrega a domicilio. Objetivo. Analizar los productos de mayor consumo mediante el servicio delivery generados ante la emergencia sanitaria en el año 2020, en la ciudad de Cuenca. Metodología. El diseño partió del paradigma de investigación positivista, con diseño de campo no experimental y transversal y apoyo documental, nivel descriptivo, la muestra utilizada fue de 50 microempresas que ofrecen el servicio en la ciudad de Cuenca. Resultados. Con mayor frecuencia con respecto al tamaño de la empresa, (62.00\%) son microempresas, por su parte los servicios prestados por las empresas de delivery son (40.43\%) de alimentos. Por otro lado, la forma en la cual se contacta para solicitar el servicio es mediante aplicaciones móviles Android y Apple (IOS) (54.00\%). El segmento de mercado que cubren con mayor frecuencia es usuarios que tienen necesidad de comprar por urgencia o conveniencia (53.13\%). El (58.00\%) de las empresas encuestadas general ingresos de $\$ 0$ a $\$ 100.000$. Las Apps están representadas por Rappi con el 19,05\% y Tipti el 18,25\%. Dentro de los estándares de calidad $(19.35 \%)$ se presta mayor importancia a las normas de bioseguridad por la presencia del COVID 19. Conclusión. El servicio de delivery se diferencia de los servicios típicos de compra convencional, porque integra una propuesta de valor para los usuarios que podrán realizar sus compras de una manera práctica, sencilla y ágil. Las mipymes cumplen un rol relevante en la economía, por su potencial de generar empleabilidad, por su aporte con el pago de tributos e impuestos al estado.

\section{Keywords:}

COVID-19,

delivery

service,

consumers,

\section{Abstract}

Introduction. During the COVID-19 pandemic, the need for mobile applications arose to find a way to bring their products to their customers without the need to expose themselves, this situation led to the birth of new ventures that last even when the pandemic is controlled, that is where companies that provide home 


\section{VPDigital}

mobile

applications delivery services are born. Target. Analyze the most consumed products through the delivery service generated in the face of the health emergency in 2020, in the city of Cuenca. Methodology. The design started from the positivist research paradigm, with a nonexperimental and cross-sectional field design and documentary support, descriptive level, the sample used was of fifty microenterprises that offer the service in the city of Cuenca. Results. More frequently with respect to the size of the company, $(62.00 \%)$ are micro-enterprises, for their part the services provided by delivery companies are $(40.43 \%)$ food. On the other hand, the way in which you contact to request the service is through Android and Apple (IOS) mobile applications (54.00\%). The market segment that they cover most frequently is users who have an urgent or convenience need to buy $(53.13 \%)$. The $(58.00 \%)$ of the surveyed companies generated revenues of $\$ 0$ to $\$ 100,000$. The Apps are represented by Rappi with $19.05 \%$ and Tipti with $18.25 \%$. Within the quality standards (19.35\%), more importance is given to biosafety standards due to the presence of COVID 19. Conclusion. The Delivery service differs from typical conventional purchase services because it integrates a value proposition for users who will be able to make their purchases in a practical, simple, and agile way. MSMEs play a relevant role in the economy, due to their potential to generate employability, due to their contribution with the payment of tributes and taxes to the state.

\section{Introducción}

El mes de marzo de 2020, Ecuador y el mundo entero entro un confinamiento debido a una pandemia ( propagación mundial de una nueva enfermedad) denominada COVID-19 virus letal que cobró la vida de miles de personas alrededor de todo el planeta, provocó además que cientos de empresas y negocios pequeños se vieran obligados a cerrar sus puertas generando con ellos pérdidas, despidos y en muchos de los casos el quiebre de las mismas, durante el confinamiento muchos buscaron maneras de sobresalir a la difícil situación económica que se presentaba, surgieron varios emprendimientos, y los pocos negocios buscaban la manera de llevar sus productos a sus clientes sin la necesidad de exponerse a esta pandemia, esta situación nos llevó a analizar también posibles emprendimientos que perduren aun cuando la pandemia sea controlada, es ahí donde nace 
realizar la presente investigación sobre el servicio delivery, generados ante la emergencia sanitaria.

La reducción de movilidad aceleró el número de usuarios de aplicaciones como Tipti, Rappi, Uber y Pedidos Ya, Picker y direcciones web de pedidos como: www.coralhipermercados.com, www.fybeca.com, siendo las mismas nuestro caso de estudio. Estas empresas durante y postpandemia tuvieron que mejorar para lograr satisfacer a las personas que demandaban sus servicios, al igual que se tuvo que crear nuevos nichos de mercado para llegar a las diferentes partes del país.

La pandemia del COVID-19, ha dado lugar a nuevas pautas de utilización y al afianzamiento de la cadena de valor de las ventas, esto implica una teoría empresarial que sirve para estimar el valor de los productos o servicios disponibles para la venta, que produce una empresa, habitualmente, compuesta por eslabones, en materia de compras, almacén, diseño, producción, ventas, inversiones, entre otros. La pandemia si bien ha afectado la salud y variadas actividades económicas de los sectores productivos empresariales, también estimuló la creatividad y el desarrollo de soluciones de negocios que contribuirán al crecimiento del servicio delivery.

El virus según Becerra et al. (2021c), ha hecho que la rutina diaria de las personas y negocios cambien hacia una jornada de estricta seguridad, distanciamiento y cuidado. Así mismo algunas empresas modificaron sus modelos de negocios, con el objetivo de continuar brindando sus productos y/o servicios. De allí que una buena parte de negocios al ver que no podían cubrir la demanda de forma presencial optaron por trabajar de forma online, usando principalmente las redes sociales como Facebook, Instagram y, WhatsApp, y sitios web y el servicio delivery.

La presente investigación, hace referencia al servicio delivery en la ciudad Santa Ana de los cuatro ríos de Cuenca, que tienen como objetivo el servicio delivery es el que ofrecen las empresas para hacer las entregas a domicilio de los productos que los clientes compran vía online o por medios móviles telefónicos, como dinámica de la productividad. Cabe destacar que este tipo de actividades ha tomado un gran espacio en la economía a nivel local, regional, nacional y mundial en estos tiempos, una buena parte del comercio se ha realizado de forma electrónica ya que resulta más fácil adquirir los productos de esta manera, por la comodidad pues evita al consumidor salir a buscar en tiendas físicas y también es un ahorro de tiempo; entre los productos y servicios más requeridos son alimentos, supermercados, farmacias, licoreras, lavanderías, regalos, artículos y herramientas menores.

El servicio delivery todavía es joven y se está formando, y el futuro se ve brillante para las empresas que se deciden a conducir sus negocios en línea, solo hay que mirar a las 
empresas como Amazon.com y eBay.com, como se ha posesionado en el mercado online y las utilidades y beneficios que estas han generado.

Este trabajo es parte del proyecto de investigación denominado "La importancia de la información contable y financiera ante la incertidumbre del COVID-19 en las MiPymes en el entorno de los negocios en la región 6", el mismo que se justifica porque contribuye desde una perspectiva teórica académica a dar salida a la importancia de los modelos de negocios del servicio delivery en las micro, pequeñas, y medianas empresas en tiempos de pandemia.

\section{Metodología}

Sobre la base de nuestra investigación se detalla cómo se sistematiza el trayecto metodológico que se utilizó para llevar a cabo el estudio, parte del paradigma de investigación positivista, con diseño de campo no experimental y transversal con apoyo documental, el nivel de la investigación fue la descriptiva, al respecto Hurtado (2010) explica que:

Su propósito es exponer el evento estudiado, haciendo una enumeración detallada de sus características, de modo tal que en los resultados se pueden obtener dos niveles de análisis, dependiendo del fenómeno y del propósito del investigador, es un nivel más elemental, en el cual se logra una clasificación de la información en función de las características comunes, y un nivel más sofisticado en el cual se ponen en relación los elementos observados (p. 73).

En este apartado de la investigación corresponde examinar algunos aportes teóricos sobre emprendimiento, se utilizó para llevar a cabo el estudio, indicando el paradigma de investigación positivista, tipo de investigación diseño de campo no experimental y transaccional o transversal con apoyo documental. Según Tamayo (2003) establece que:

En la investigación de campo, los datos se recogen directamente de la realidad, razón por lo que se les denomina primarios, lo cual según el precitado autor su valor radica en que permite cerciorarse de las verdaderas condiciones en que se han obtenido los datos, lo cual facilita su revisión o modificación en caso de surgir dudas (p.65).

El nivel de investigación fue la investigación descriptiva. Según Arias (2016), "la investigación descriptiva consiste en la caracterización de un hecho, fenómeno, individuo o grupo, con el fin de establecer su estructura o comportamiento" (p.24).

Se corresponde, por tanto, con el nivel descriptivo en función del objetivo general planteado, la población estuvo estructurada por micro, pequeñas y medianas empresas de la ciudad de Cuenca, de una población de 200, se determinó una muestra estratificada 
mediante una muestra aleatoria simple de $\mathrm{n}=50$, la validez de contenido fue realizado mediante el juicio de tres expertos con experiencia en el área de emprendimiento, contabilidad y metodología.

Por su parte Becerra et al. (2021d), fue sustancial la utilización de la herramienta estadística para el desarrollo de la investigación, recurriendo a la estadística descriptiva, lo que permitió la recolección, organización, análisis y presentación de datos con el apoyo de cuadros, tablas, figuras y los resultados obtenidos de la aplicación de los cuestionarios en relación con los objetivos planteados del presente estudio, mediante el software libre SPSS, versión 26.

El análisis de los datos es una parte esencial de toda investigación, en tal sentido Hernández et al. (2016), sostienen que "el procedimiento que se sigue para analizar los datos consiste en: una vez que los datos se han codificado, transferido a una matriz, guardado en un archivo y "limpiado" los errores, el investigador procede a analizarlos utilizando un programa computacional” (p. 272). En base a lo cual se procedió a inspeccionar, limpiar y transformar datos con el objetivo de resaltar información útil, lo que sugiere conclusiones, y apoyo a la toma de decisiones.

Posterior a los procedimientos que condujeron a reunir datos con el propósito específico de estructurar el análisis del servicio delivery, se procedió a revisar los datos o respuestas obtenidas mediante Google forms. Las respuestas a los cuestionarios se recopilan de forma automática y ordenada en formularios, con figuras y datos de las respuestas en tiempo real, que fueron procesados, analizados e interpretados, considerando el uso de la triangulación, validez, confiabilidad y contrastación de los instrumentos.

\section{Marco teórico}

El marco teórico referencial comprende aspectos teóricos, conceptuales, legales, situacionales de la realidad que forma parte de la presente investigación, garantizando de esta manera la integración e interrelación de la teoría con la metodología y objetivos de investigación.

Según el Observatorio Sectorial de la Restauración de DBK (2020), el servicio de "delivery" creció un $26 \%$ durante 2020. Una de las grandes ventajas de este servicio, además, es que promueve el logro de ingresos complementarios por parte de los establecimientos de restauración organizada. Así, los datos del observatorio apuntan a que, durante el mismo período, este servicio ha impulsado las ventas de los establecimientos de restauración rápida un 7,3\%. En términos de valor, las ventas a domicilio se incrementaron un 26\%, cifrándose en 605 millones de euros y representando ya el $16 \%$ de la facturación del sector de comida rápida, tras las secuelas que está dejando la pandemia del COVID-19. 
Según la Comisión Económica para América Latina y el Caribe- CEPAL (2019), El conocimiento sobre cómo las empresas de América Latina y el Caribe usan Internet para involucrarse en el comercio, y en particular los desafíos que enfrentan para adoptar y usar el comercio electrónico para exportar bienes y servicios, es aún bastante incipiente, en consecuencia el propósito de este documento es analizar los desafíos que enfrentan las empresas de América Latina y el Caribe en su comercio electrónico, mediante la explotación de datos de encuestas que cubren a más de 1.430 empresas de la región, junto con datos económicos y de consumo, así como entrevistas con firmas digitales. También propone algunas recomendaciones de políticas sobre cómo las economías regionales pueden trabajar juntas para potenciar el servicio delivery y comercio electrónico transfronterizo en la región.

Tal como lo señala Euromonitor International (2020), tanto el segmento de la comida rápida como el de la industria delivery, han logrado alzas importantes en el mercado con tasas de crecimiento anuales que rozan el $20 \%$. El secreto de esta popularidad se debe a las promociones proporcionadas por las aplicaciones delivery en conjunto con la eficacia del servicio, donde se destaca su crecimiento, cuando las ventas crecieron en un $92 \%$, teniendo que reforzar los servicios para dar abasto, aunque se esperaba que por la crisis esta tendencia de compra iba a decrecer, las estimaciones de la demanda han logrado invertirse.

En el caso del Ecuador según revista Ekos, La Cámara Ecuatoriana de Comercio Electrónico (2021), el comercio electrónico en Ecuador alcanzó un volumen de negocio de USD 2.3 mil millones, lo que supone un crecimiento de USD 700 millones (43,75\%) frente al 2019. Para el 2021 se estima un crecimiento no menor a un doble dígito. Igualmente sostiene que en el año 2020 marcó un antes y un después en el comercio online. El contexto obligó a las marcas y a los profesionales del sector a realizar esfuerzos para adaptarse a toda velocidad a la nueva realidad y al nuevo consumo, es así como uno de cada tres compradores eligió por primera vez el canal online para generar sus compras, además reportó un crecimiento del $800 \%$ en visitas a los sitios webs y un $44 \%$ en órdenes en comparación con 2019, según las últimas estadísticas de la Cámara Ecuatoriana de Comercio Electrónico (CECE).

Es importante que todos aquellos ecuatorianos que estén relacionados con el uso del comercio electrónico y el servicio delivery, nos encontremos con total conocimiento sobre la Ley de Comercio Electrónico, Firmas y Mensajes de Datos (2014), que sostiene la necesidad de impulsar el acceso de la población a los servicios electrónicos que se generan por y a través de diferentes medios electrónicos y que mediante el uso del servicio de redes electrónicas, incluida la internet, se establecen relaciones económicas y de comercio, y se realizan actos y contratos de carácter civil y mercantil. 
En tal sentido la ciudad de Cuenca se ha sumado a este modelo de intercambio. Becerra et al. (2021d), sostiene que los canales convencionales de compra, mediante las plataformas tecnológicas y las aplicaciones móviles Android y Apple (IOS), de allí que se puede notar la continua penetración del comercio electrónico y el servicio delivery, por medio de las compras por internet, esta realidad obliga a los negocios locales a desarrollar nuevos modelos de ventas, ya que la carrera tecnológica en esta materia seguirá creciendo y este apogeo tendrá un precio alto para los dueños de comercios que no apliquen herramientas tecnológicas, esto lo reconoce inclusive las cámaras de la producción de la ciudad de Cuenca.

Según Becerra et al. (2021c), confirma que en la ciudad de Cuenca-Ecuador, se considera como una zona empresarial, se ve un alto interés en participar dentro de las estrategias del e-comerce, entre las cuales se encuentra el servicio delivery, lo cual son acciones que, en el país y la región, es algo bastante nuevo, pero que a razón de la situación se ha tenido que implementar, lo que ha creado alternativas de comercio mucho más efectivas y cómodas.

La palabra delivery, es un vocablo ingles que significa entrega, es decir es el proceso logístico de envío que antes generalmente lo realizaba el consumidor y ahora lo puede realizar la empresa que da el servicio o produce por un costo adicional o sin él.

Por su parte, la entrega a domicilio o la entrega directamente al cliente surgió en la década de los ochenta en territorios de habla inglesa y luego traído al Ecuador en el año 2014, la venta de los productos y servicios ante cada vez más agitados consumidores.

Este tipo de actividades es realizado por llamadas telefónicas y directamente a la empresa que realizaba la venta del producto o servicio. La contrariedad surge, cuando no todas las empresas poseen este tipo de servicio y por otro lado no es fácil conseguir el número telefónico de dicha empresa.

En el tiempo actual el servicio de entrega a domicilio tomó fuerza a raíz de la pandemia del Covid-19, es a partir del año 2020, que surgen las páginas web a través del internet, cada empresa que vendía algo ofrecía su servicio de entrega por página web, es decir comenzaron a dar el servicio de intermediación entre el cliente y el productor, así hay empresas que tercerizan este servicio, buscando siempre satisfacción del cliente, buscando siempre para que en el futuro lo recomienden o vuelvan a solicitar el servicio. A ello se agrega la página web, en donde no solo está un producto, sino existen muchas marcas para un mismo producto. Pero el nuevo cliente ante la emergencia sanitaria, recién se acostumbraba a hacerlo a través de internet.

En la actualidad con la llegada del smartphone que agrupa el teléfono móvil, que es un dispositivo de cómputo con una línea de internet rápida y a un solo toque, hizo que este 


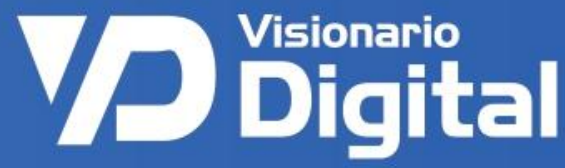

tipo de servicio creciera exponencialmente no solo en el mundo, sino particularmente en la ciudad de Cuenca.

En consecuencia, a comparación de una página web, la aplicación móvil es más fácil no solo por la rapidez de la red sino porque es posible acceder a través del celular la geolocalización, la hora y adicionalmente el cliente se lleva la satisfacción de tener todo a un toque y la respuesta de este es rápida.

La ciudad Santa Ana de los cuatro ríos de Cuenca es un mercado para los servicios de "delivery" relativamente nuevo porque en el mercado han ido apareciendo modelos de negocio donde el servicio de intermediación está tomando relevancia en la masificación de teléfonos inteligentes.

\section{Resultados}

Para el procesamiento y análisis de la información proporcionada por las micro, pequeñas y medianas empresas (MiPymes), en el presente trabajo de investigación, fue necesario acudir a la técnica de la encuesta y el instrumento del cuestionario, en un total de 8 ítems.

\section{Figura 1}

Tamaño de la empresa, según el número de empleados

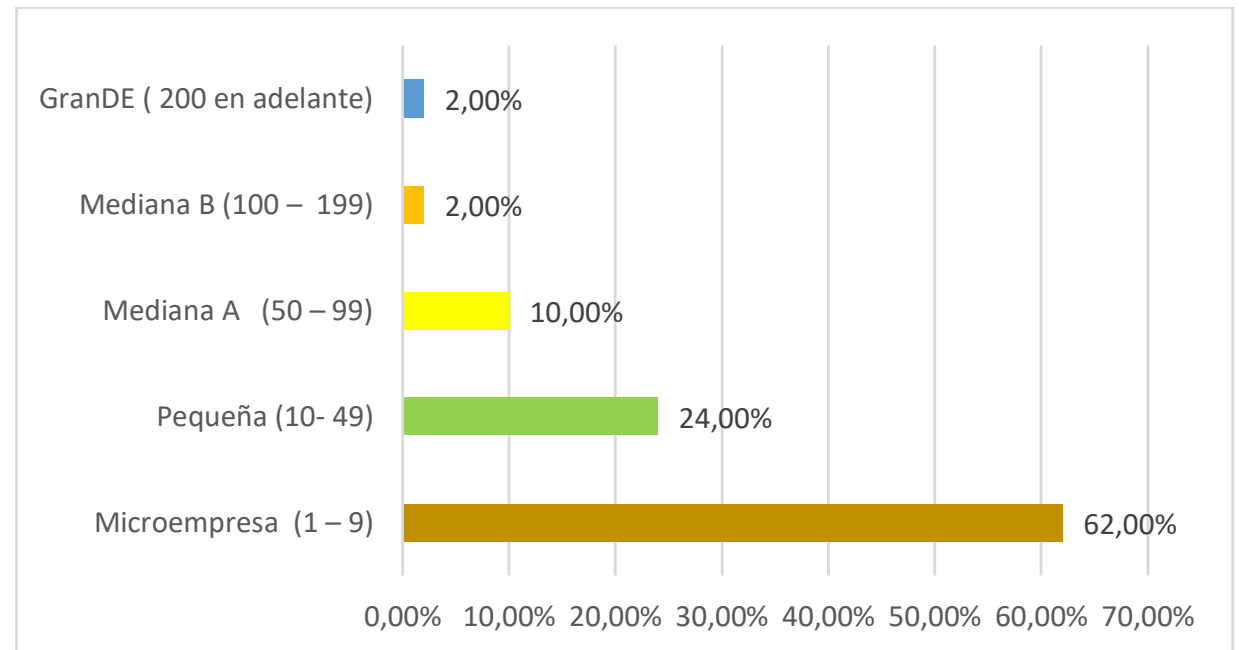

Fuente: Elaboración propia (2021). Tomado del proyecto de investigación la importancia de la información contable y financiera ante la incertidumbre del COVID-19 en las MiPymes en el entorno de los negocios en la región 6.

De acuerdo al cuestionario aplicado el tamaño de las empresas según el número de empleados en la ciudad de Cuenca, los resultados determinaron que el $62 \%$ de las empresas de delivery, es decir, que consiste en prestación de servicios que ofrecen para hacer las entregas a domicilio de los productos que los clientes compran vía online ya sea atreves de un computador, tablet o teléfono inteligente, este tipo de empresas suelen estar 


\section{VP告igigitital}

administradas por sus propios dueños y algunas veces están formadas por un grupo familiar, que tienen como premisa buscar el crecimiento de la empresa de forma conjunta.

El $24 \%$ pertenece a pequeñas empresas, que son empresas de producción, que se encuentran constituidas de entre 10 y 49 trabajadores, sus ingresos anuales lo constituyen entre de \$100.001 y 1.000.000, conforme (La Superintendencia de Compañías, 2021).

El $10 \%$ de las empresas encuestadas, pertenece a las medianas empresas, que lo constituyen entre 50-99 empleados, sus ingresos anuales están dados entre \$1.000.001 y 5.000.000, en tal sentido son sociedades o de un solo propietario, tienen libertad de desarrollar cualquier tipo de actividad, bien sea de producción, comercialización o prestación de servicios, su objetivo es lograr rentabilidad.

Mientras que el $2 \%$ pertenece a las medianas empresas que lo constituyen aquellas unidades de producción, se caracterizan por que tienen de 50 a 199 trabajadores y un valor de ventas o ingresos brutos anuales entre millón (USD 1.000.001,00) y (USD 5.000.000,00) dólares de los Estados Unidos de América.

Por último, las grandes empresas, también con el 2\%, tienen más de 200 trabajadores, además sus ingresos son superiores a los $\$ 5.000 .001,00$, por tales razones desde una perspectiva económica, la empresa se caracteriza como una entidad autónoma de producción de bienes o servicios, en la que se integran de forma coordinada diversos medios productivos a saber: la fuerza de trabajo, recursos materiales, recursos económicos, recursos tecnológicos, elementos materiales e inmateriales, bajo la dirección del empresario.

\section{Figura 2}

Clase de empresa a la que pertenece

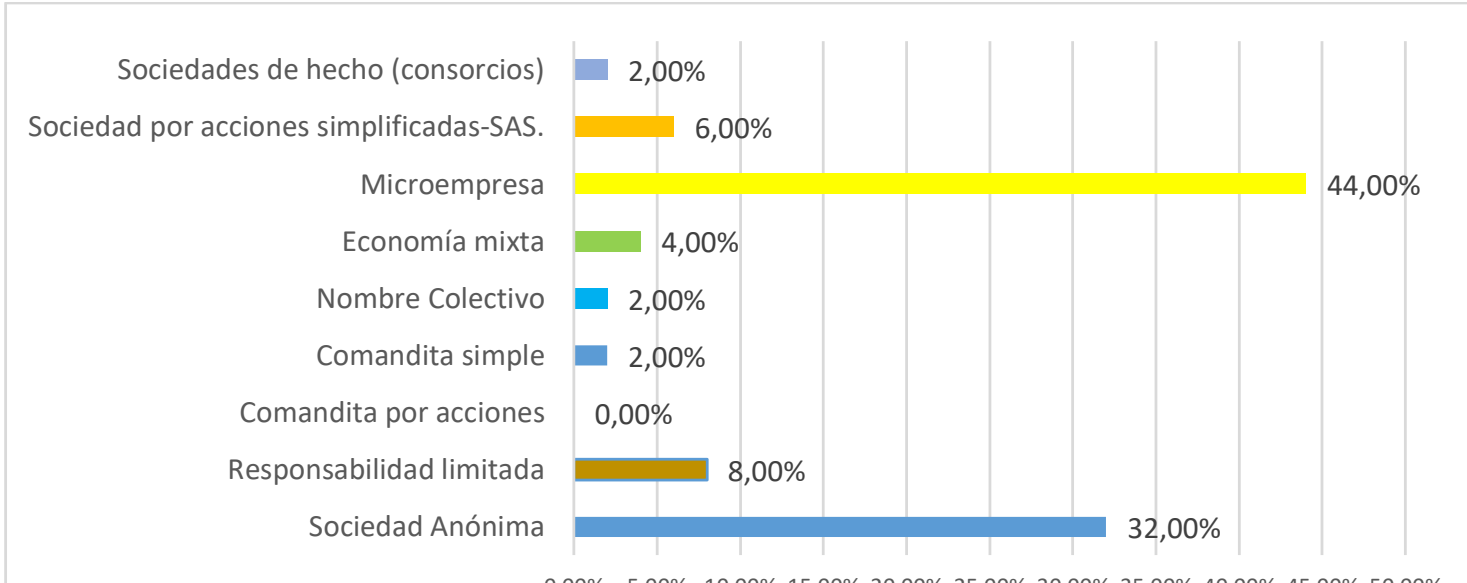

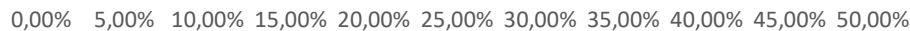

Fuente: Elaboración propia. Tomado del proyecto de investigación la importancia de la información contable y financiera ante la incertidumbre del COVID-19 en las MiPymes en el entorno de los negocios en la región 6. 
Según los resultados del cuestionario aplicado el $44 \%$ pertenece a la microempresas, puede ser operado por una persona natural, una familia, o un grupo de personas de ingresos relativamente bajos, el propietario ejerce un criterio independiente sobre los servicios, mercados y precios y además constituye una importante fuente de ingresos para la familia, en general comprenden a organizaciones económicas populares, tales como, los emprendimientos unipersonales, familiares, barriales, comunales, las micro unidades productivas, los trabajadores a domicilio, los comerciantes minoristas, los talleres y pequeños negocios, que integran la economía popular y serán promovidas con criterio de solidaridad creando y fomentando programas de inclusión, reconocer, fomentar y fortalecer la economía popular y solidaria y el sector financiero popular y solidario en su ejercicio y relación con los demás sectores de la economía y con el Estado; y potenciar las prácticas de la economía popular y solidaria que se desarrollan en las comunas, comunidades, pueblos y nacionalidades, y en sus unidades económicas productivas para alcanzar el Sumak Kawsay, conforme consta en la Ley de Encomia Popular y Solidaria, en su artículo 3 (Ley Orgánica de Economía Popular y Solidaria, 2018).

El 32\% pertenece a las sociedades, son compañías con personería y personalidad jurídicas creadas legalmente de acuerdo con los parámetros de la ley compañías, bajo el control de la superintendencia de compañías, valores y seguros, de acuerdo con la estructura de control establecida por el gobierno central.

El 8\% pertenece a compañías de responsabilidad limitada, la que se contrae entre dos o más personas, que solamente responden por las obligaciones sociales hasta el monto de sus aportaciones individuales y hacen el comercio bajo una razón social o denominación Cía. Ltda.

El 6\% pertenece a sociedades por acciones simplificadas, es un tipo de compañía que se constituye por una o varias personas naturales o jurídicas, mediante un trámite simplificado sin costo. Tiene como objetivo impulsar la economía mediante la formalización de los emprendimientos, constituyéndolos en sujetos de crédito y con ello ampliar sus procesos productivos (Superintendencia de Compañías, 2021).

El resto de las variables se consideran en menor proporción, así las de la economía mixta el $4 \%$, la comandita simple, nombre colectivo y sociedades de hecho, estas tres últimas con el $2 \%$ cada una, por ello la figura de la sociedad mercantil ha adquirido y adquiere gradualmente una mayor notoriedad económica. En la práctica, la sociedad, como organización empresarial, se atribuye cada vez más al empresario individual debido, fundamentalmente, a que, desde el ámbito de la sociedad, se pueden afrontar más fácilmente y con menos riesgo empresas que requieren un alto grado de inversión o de recursos, por consiguiente, las sociedades se constituyen en el principal motor de la vida económica del país. 


\section{VPDigital}

\section{Figura 3}

Servicios requeridos por parte los usuarios

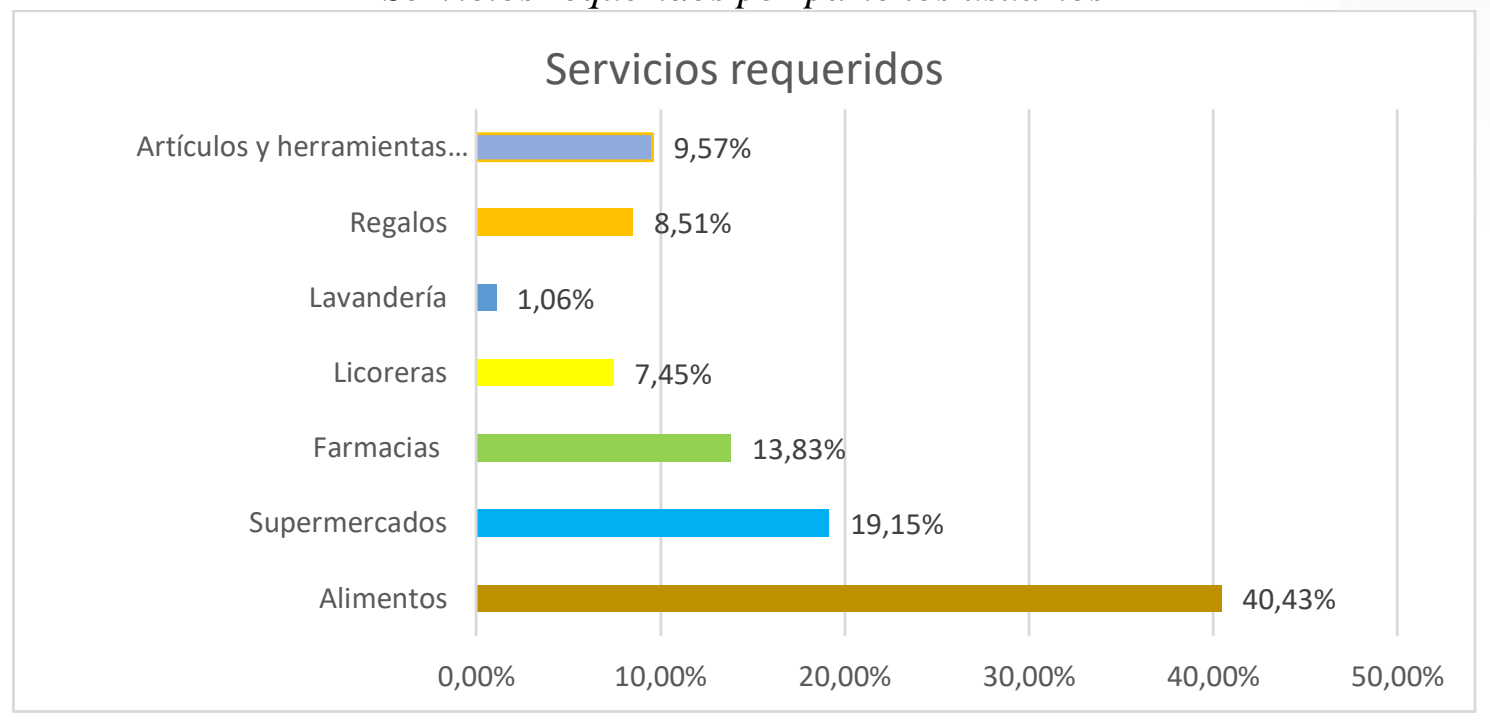

Fuente: Elaboración propia (2021). Tomado del proyecto de investigación la importancia de la información contable y financiera ante la incertidumbre del COVID-19 en las MiPymes en el entorno de los negocios en la región 6.

Según la figura 3, durante la pandemia observamos que una de las actividades que se incrementaron fue la adquisición de alimentos que llega a un porcentaje de $40.43 \%$ seguido de un $19.15 \%$ proveniente de los supermercados que si bien es cierto se vieron afectados por las distintas restricciones tanto en aforos permitidos como horarios restringidos, seguido de la industria farmacéutica en un porcentaje de $13.83 \%$, es en esta pandemia provocada por el COVID-19, que los hospitales públicos y privados, laboren al límite de su capacidad y al existir mayor demanda se da escasez de medicamentos como por ejemplo azitromicina, paracetamol, aspirinas, complejo B, entre otras las personas que optan por recurrir a farmacias, la adquisición de artículos y herramientas ocupa un porcentaje de $9.57 \%$ con el confinamiento quizás la mayoría de personas optaron por buscar actividades en las que se puedan distraer entre ellas estuvo el de hacer reparaciones y a quienes tenían sus casas con cuartos extras adecuaciones que podrían servir para el ejercicio de alguna actividad comercial, la gente durante la pandemia analizó sus talentos y en ello una forma de adquirir ingresos extras; el hacer adornos o arreglos para regalos está de auge con un porcentaje de $8.51 \%$ seguido del negocio de licores con un porcentaje de $7.45 \%$, aquí vemos que los negocios de lavandería sufren una caída en sus ingresos debido no solo al hecho de que ya la mayoría de personas cuentan con una lavadora en sus hogares a más de eso al estar muchos en confinamiento o teletrabajo las amas de casa tienen más tiempo para dedicarse a las labores del hogar es por ello que las lavanderías apenas llegan a un $1.06 \%$. 


\section{Figura 4}

Contacto para los servicios de delivery

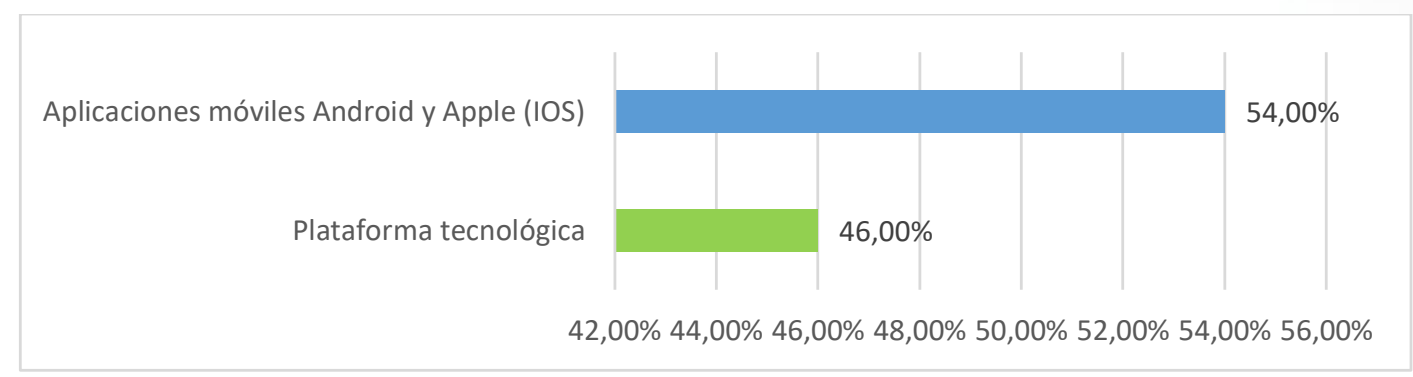

Fuente: Elaboración propia (2021). Tomado del proyecto de investigación la importancia de la información contable y financiera ante la incertidumbre del COVID-19 en las MiPymes en el entorno de los negocios en la región 6.

Según los resultados del cuestionario aplicado el 54\% pertenece a las aplicaciones móviles Android e IOS esta aplicación nos facilitan es un programa que se puede descargar y al que se puede acceder directamente desde su teléfono o desde algún otro aparato móvil, se requiero de un Smartphone o algún otro dispositivo móvil que tenga acceso a internet, de allí que se han puesto estos medios de comunicación y de comercio a la vanguardia en el mercado ya que nos permite únicamente con dar un clic, desde la comodidad de nuestro hogar acceder a un sin número de productos y servicios siendo esta la principal fuente de acceso al servicio de delivery el cual ofrecen todas las empresas para hacer las entregas a domicilio de los productos que los clientes compran vía online, así mismo tenemos el $46 \%$ en plataformas tecnológicas las cuales son de similar uso mediante un aparato tecnológico con acceso a internet como pueden ser las redes sociales donde se puede acceder publicar o vender de forma gratis y sin costo.

\section{Figura 5}

Segmento de mercado

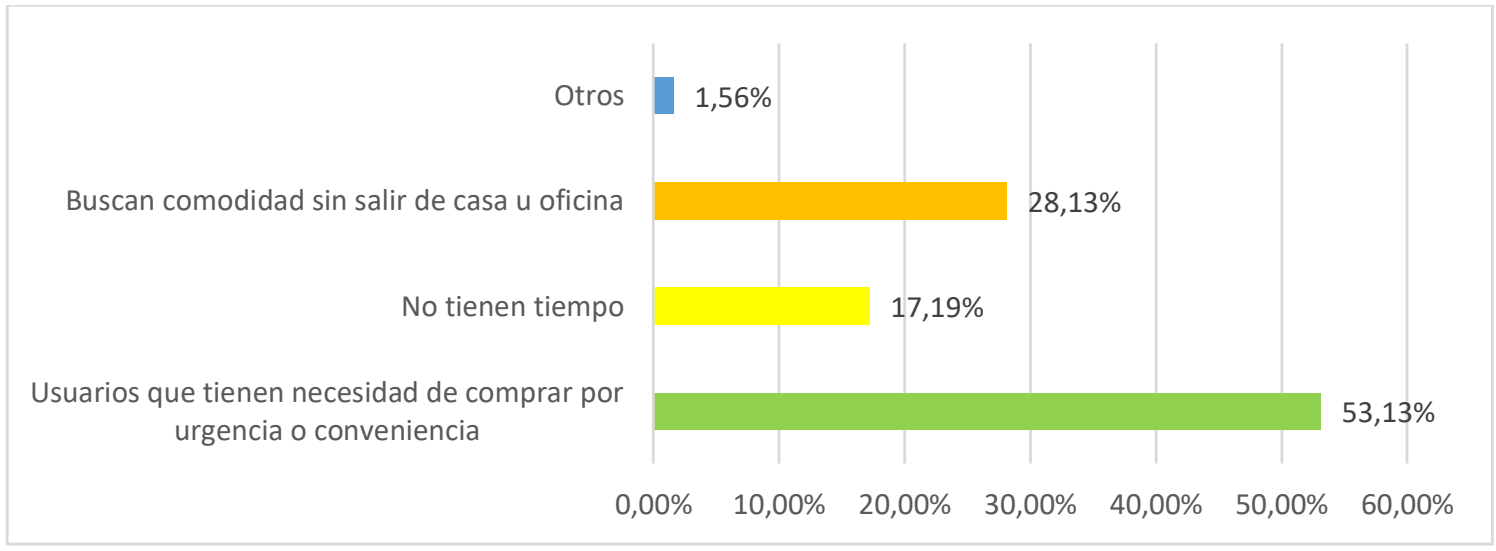

Fuente: Elaboración propia (2021). Tomado del proyecto de investigación la importancia de la información contable y financiera ante la incertidumbre del COVID-19 en las MiPymes en el entorno de los negocios en la región 6. 
Según, los resultados de cuestionario el 53,13\%, los usuarios que tiene la necesidad de comprar por urgencia o conveniencia siendo la más representativa en nuestro estudio, ya que al momento de generar el subconsciente, al momento de adquirir un servicio o producto lo primero que se nos viene a la mente es adquirir mediante una plataforma digital y que nos la entreguen en la comodidad de nuestro hogar, dentro de este porcentaje nos encontramos todos nosotros ya que siempre buscamos cada vez comodidad al momento de comprar por urgencia y al utilizar el servicio de delivery se optimiza el tiempo y costo, seguido de personas o usuarios que buscan comodidad con un $28,13 \%$ siendo un potencial más para el uso de delivery ya que actualmente existe muchas más promociones, si accedemos mediante servicio a domicilio que acercarnos a comprar directamente inclusive obteniendo descuento y promociones para próximas compras, por su parte el 17,19\% sostuvieron que compran utilizando el servicio de delivery porque no tienen tiempo, ya sea porque su trabajo no les permite o por múltiples actividades que realizan, en tanto que el 1,56\% sostiene que se debe a otros, en definitiva es un recurso que representa beneficios para restaurantes, farmacias o cualquier negocio que quiera destacarse de otro. Del mismo modo, ofrece servicios de valor alternos, como la posibilidad de pagos con tarjeta aún a domicilio, aumentando la posibilidad de que los consumidores, al ver la cantidad de posibilidades de la marca, la prefieran y piensan en ella antes que en cualquiera que ofrezca los mismos bienes y/o servicios.

\section{Figura 6}

Ingresos generados en el 2020 en empresas que presta servicios delivery

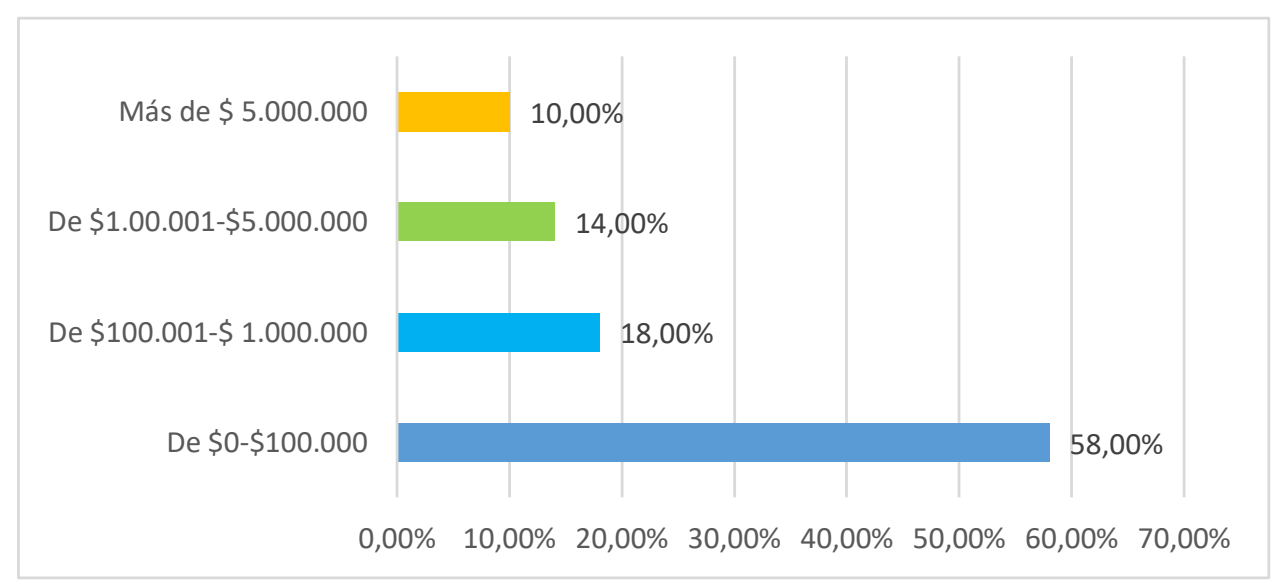

Fuente: Elaboración propia (2021). Tomado del proyecto de investigación la importancia de la información contable y financiera ante la incertidumbre del COVID-19 en las MiPymes en el entorno de los negocios en la región 6.

Tras la pandemia COVID 19, según la Comunidad Andina - CAN (2021), el sector con más impacto fue el comercial, sin embargo, los productos alimenticios y de primera necesidad fueron altamente requeridos, para el desarrollo de las Pymes y grandes Empresas se vieron en la necesidad de ofrecer el servicio de delivery como medio de 
accesibilidad para sus clientes. De allí surge el sector de las microempresas quienes en su mayoría surgieron en medio de COVID-19, como estrategia tras la disminución de jornadas laborales y reducciones de personal que se presentaron fueron los más beneficiados teniendo un porcentaje de $58 \%$ de uso de la aplicación, que generaron ingresos hasta \$100.001 dólares, de la misma manera las pequeñas empresas tuvieron un porcentaje del $18 \%$ con ventas que van entre $\$ 100.001$ y a $\$ 1.000 .000,00$, las medianas empresas con el $14 \%$, con ventas que van de entre $\$ 1.000 .000$ a $\$ 5.000 .000$, y con el $6 \%$ con ventas que van más de $\$ 5.000 .000$, teniendo en cuenta que las grandes empresas tienen ahorros acumulados por la compra de grandes cantidades de bienes y entre sus ventajas está la facilidad de financiamiento que da mayor garantía a las empresas del pago de sus deudas y sus barreras de entrada son relativamente escasas debido a la gran cantidad de mano de obra.

Figura 7

Apps y Webs de delivery a domicilio

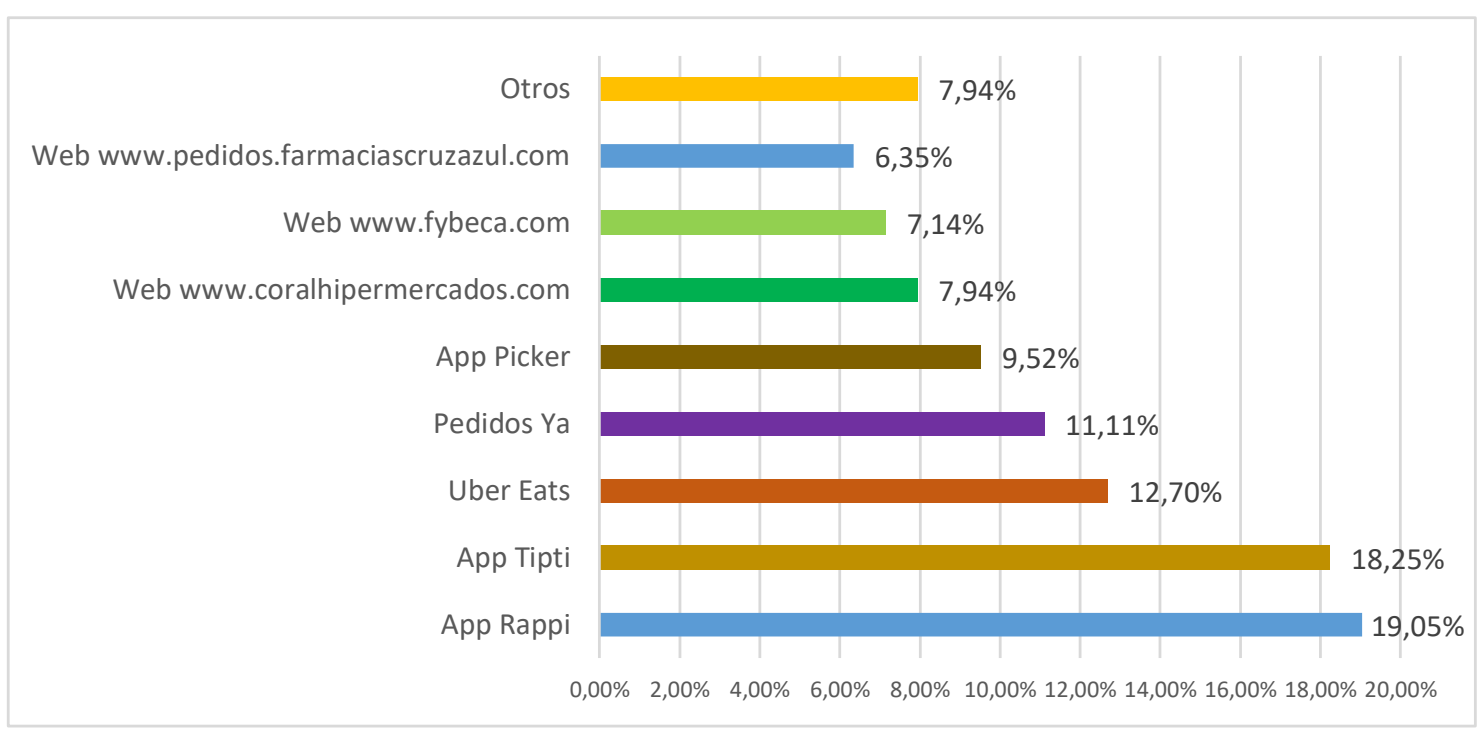

Fuente: Elaboración propia (2021). Tomado del proyecto de investigación la importancia de la información contable y financiera ante la incertidumbre del COVID-19 en las MiPymes en el entorno de los negocios en la región 6.

El análisis indicó que la App Rappi tiene el 19,05\% de aceptación, es una de las que ofrece entrega de productos a domicilio de restaurantes, compras, medicinas, licores, entre otros, tienen un servicio exprés si se necesitan pocos productos de forma rápida. Así mismo la App Tipti con el 18,25\%, es una plataforma de servicio a domicilio de los pedidos de productos de la Corporación Favorita, tanto en Supermaxi, Megamaxi, Akí, Súper Akí, Gran Akí, Akí, entre otros, así mismo Uber Eats con el 12,70\%, perteneciente al mismo grupo de la aplicación de vehículos de transporte Uber, Uber Eats está disponible en los sistemas operativos Android y iOS. Solo debe crear una cuenta en la aplicación a través 
del teléfono celular y correo electrónico. Por su parte Pedido Ya, con el 11,11\%, allí puede solicitar los servicios que se ofertan, ya sea de pedidos de restaurantes a domicilio, supermercados, farmacias, bebidas y envíos en que se pueden encontrar productos como flores, regalos, papelería, tecnología, productos para mascotas y más.

Igualmente, la App Picker, permite hacer compras en diversos locales, pago de planillas o envío de encomiendas, también hacer compras en tiendas e incluso hacer compras en mercados municipales, Está disponible en Google Play y en la Apple Store. Existen otras aplicaciones con el 7,94\%, especialmente para el servicio express, de cafeterías y restaurantes como Isnardy Restaurad Coffe, y mototax express para encomiendas.

De igual forma, existen múltiples plataformas que nos permiten acceder a una página web, entre la que tenemos la de coral hipermercados, que cuenta con el servicio a domicilio, atienden a nivel nacional de 08:30 a 19:30 de lunes a viernes. El sitio Web www.fybeca.com con el $7,14 \%$, la cadena de farmacias Fybeca recibe pedidos para domicilio a través de su sitio web, por último, la Web pedidos.farmaciascruzazul.com con el 6,35\%, de la cadena de farmacias Cruz Azul mantiene habilitado el servicio de entrega bajo pedido, a través del cual el cliente puede realizar su requerimiento desde la casa y la empresa farmacéutica lo tendrá listo para que sea retirado. Para ello deben ingresar al sitio web, donde encontrarán un botón con enlace directo al WhatsApp de la farmacia más cercana por geolocalización, para que los usuarios puedan contactarse al número celular de la farmacia, realizar su pedido validando stock y coordinar el día de retiro; para ahorrar tiempo a los clientes, evitando filas y riesgo del contagio del virus.

\section{Figura 8}

Estándares de calidad y sanidad alimentaria que exige el contexto actual

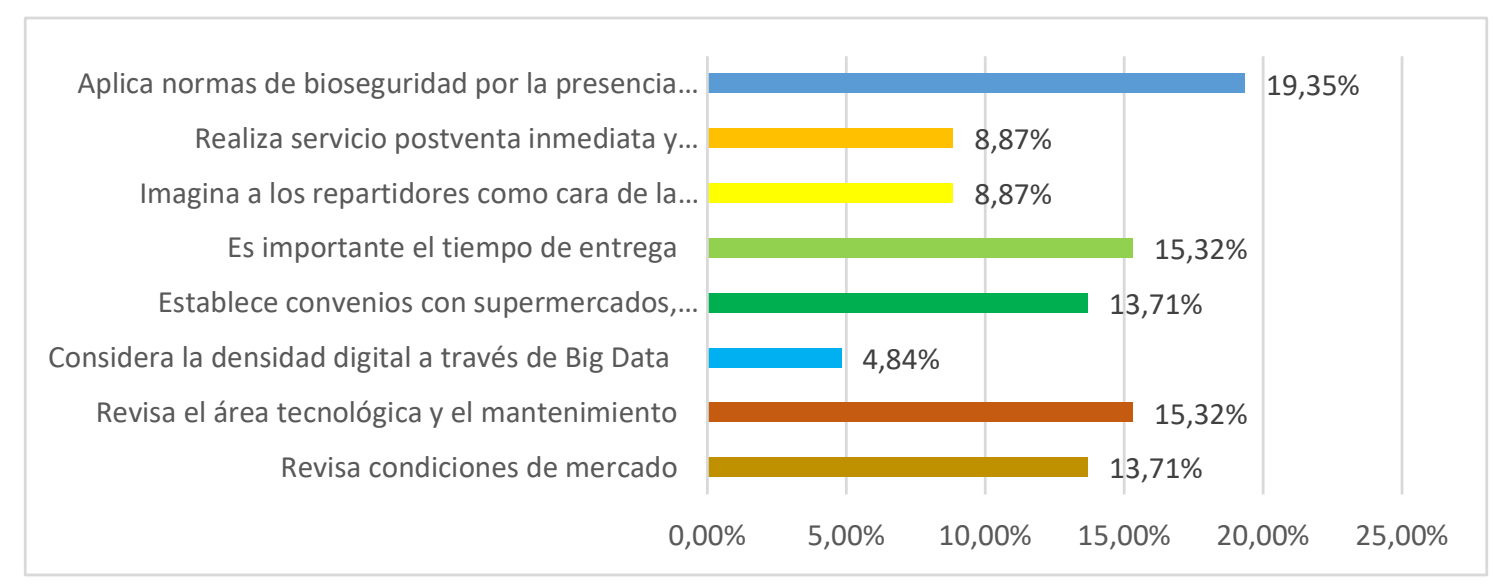

Fuente: Elaboración propia (2021). Tomado del proyecto de investigación la importancia de la información contable y financiera ante la incertidumbre del COVID-19 en las MiPymes en el entorno de los negocios en la región 6. 
Según resultados obtenidos del cuestionario aplicado se puede evidenciar que $19.35 \%$ de las empresas aplica normas de bioseguridad por la presencia de la COVID 19, es decir para ellos lo principal es la desinfección de los productos a entregar, mientras que el $15.32 \%$ considera importante el tiempo de entrega, seguido también del 15.32\%, revisa el área tecnológica y el mantenimiento están concentrados en la recepción de pedidos en sus respectivas plataformas, en cuarto lugar con el $13.71 \%$ revisa las condiciones de mercado concentran su atención en la competencia entre empresas, por ejemplo: el número de competidores, costos y tiempos de entrega, entre otros, también el $13.71 \%$ establece convenios con supermercados, farmacias y otros, el $8.87 \%$ realiza servicio de postventa inmediata y personalizada, el $8.87 \%$ de los directivos de las empresas imagina a los repartidores como imagen de la compañía y el $4.84 \%$ considera la densidad digital a través de Big Data. "Big Data son los grandes volúmenes de datos que se emiten segundo a segundo por un like, tweet o sencillamente la transacción que acaba de realizar en el supermercado" (Aldana, 2018).

\section{Discusión}

La presencia de la pandemia del COVID-19, en el país no sólo está conmoviendo la salud de los habitantes ecuatoriano, sino en varios contornos y latitudes de la humanidad como son la educación, salud, transporte público, seguridad, trabajo, el comercio, la economía, la incertidumbre está flotando que no se sabe cuál será el pico de la pandemia en el país y cuáles serán los efectos en materia de actividad y empleo, por ello Becerra et al. (2021c), sostienen que nadie contaba con que llegaría un virus que forzaría a miles de personas a recluirse en sus hogares y cambiar considerablemente sus hábitos de consumo y la forma en que realizan sus compras, mediante el uso de las aplicaciones móviles para pedir comida, medicamentos, compras de víveres, entre otros, se ha tornado en algo natural para el consumidor que vive en la contingencia causada por la epidemia, sin embargo, no se ha convertido en un hábito ni ha generado dependencia o adicción alguna, por ello se analizó a los consumidores, que conforme Reyes et al. (2021), considera a la apps móviles, como las ganadoras durante el coronavirus, y los hábitos de los consumidores se han modificado de una manera drástica en cuanto al consumo de alimentos se refiere, de allí que el servicio delivery en la ciudad de Cuenca ha tenido en estos últimos tiempos un crecimiento exponencial.

Según Becerra et al. (2021c), se ve un alto interés en participar dentro de las estrategias del e-commerce, lo cual son acciones que, en el país y la región, es algo bastante nuevo, pero que a razón de la situación se ha tenido que implementar, lo que ha creado alternativas de comercio mucho más efectivas y cómodas.

Así mismo Becerra \& Calle (2020), la información recogida, da cuenta que el comercio electrónico elimina la necesidad de una tienda física, por tanto, la empresa puede ahorrar 
dinero en arrendamiento, mantenimiento, servicios públicos y contratación de personal. Además, dado que no hay límite para la cantidad de artículos que se pueden vender en línea, las existencias de una tienda virtual pueden expandirse de manera exponencial en un modelo de comercio electrónico, y puede permanecer abierta las 24 horas para que los consumidores puedan comprar sus productos a la hora que deseen.

Es conveniente señalar que todos aquellos ecuatorianos que estén relacionados con el uso del comercio electrónico, nos encontremos con total conocimiento sobre La Cámara Ecuatoriana de Comercio Electrónico- CECE. (2014), que sostiene la necesidad de impulsar el acceso de la población a los servicios electrónicos que se generan por y a través de diferentes medios electrónicos y que, a través del servicio de redes electrónicas, incluido el internet, se establecen relaciones económicas y de comercio, y se realizan actos y contratos de carácter civil y mercantil.

La Cámara Ecuatoriana de Comercio Electrónico- CECE. (2021), sostiene que es conveniente destacar la educación a la oferta, que implica profesionalizar la misma que existe en comercios en línea, sean productos y servicios, o por cualquier canal no presencial; otorgar conocimientos específicos para brindar una mejor experiencia, igualmente es necesario entender la demanda, ya que las empresas deben identificar y diferenciar los productos que los usuarios prefieren comprar vía online y cuáles prefiere comprar de manera presencial; esto permitirá tener un panorama más claro, difundir los beneficios de comprar online y realizar los ajustes necesarios para brindar una mejor experiencia e interacción online, en torno a fortalecer la relación público-privada, es necesario estrechar alianzas entre la empresa privada con el Gobierno para efectuar una mejor toma de decisiones y al diseño de estrategias que sean beneficiosas para ambas partes y para el país a nivel de tributos y en condiciones de mercado.

Así mismo Becerra et al. (2021c), es importante señalar que, a través del portal web de la Cámara Ecuatoriana de Comercio Electrónico, se coloca a disposición de sus usuarios productos de primera necesidad, comida, bebidas; además su contenido es gratuito para acceder a webinars (clases en línea), sumado a una mínima parte de artículos electrónicos, entre otros, generando un atractivo que permite ingresar a las arcas fiscales algo de impuestos por la facturación del sitio.

Según Becerra et al. (2021a), en su artículo medidas financieras en el entorno de los negocios, para minimizar los efectos de la pandemia COVID-19, nutren sobre la importancia a la implementación o mejoramiento de las tiendas online, es decir tiendas virtuales o tiendas electrónicas, un tipo de comercio que usa las bondades de la tecnología y el comercio electrónico como herramientas esenciales para realizar sus transacciones a través de un sitio web o una aplicación conectada a internet. 
De igual forma Becerra et al. (2021b), en el artículo la importancia de la información contable ante la incertidumbre del Covid-19, en las MiPymes en el entorno de los negocios en la región 6, en su análisis de los ingresos o por actividades ordinarias, de las empresa, mencionan que se incorporó el comercio electrónico, es decir decidieron tratar de acoplarse a la nueva forma de ventas a causa del COVID 19, de allí que los negocios han sido afectadas debido a la situación actual de pandemia mundial y han realizado ventas a través de internet lo cual ha hecho que se avance en materia de comercio en línea.

La información recogida permitió determinar que las apps, llamadas aplicaciones móviles en realidad, se encuentran desde un inicio en el mercado: primero fue exclusivo de las computadoras, después con la llegada de las tablets y los smartphones las empresas se dirigieron hacia las nuevas tecnologías, considerando que las apps no son más que herramientas de software escritas en distintos lenguajes de programación, según el desarrollador que la programe, caracterizados por ser ventajosas, dinámicas y factibles de instalar y manejar. Actualmente existen aplicaciones de diversos tipos, que implica noticias, juegos, redes sociales, mensajería instantánea, deportes, idiomas, temas profesionales, comercio, servicios, entre otros.

Como es lógico, el negocio de "delivery" mediante un teléfono inteligente está en pleno desarrollo en el Ecuador, ya que en la actualidad están ingresando nuevas empresas que no solo se sitúan en el sector comidas sino también en el sector comercial como prendas de vestir, sector bisutería, calzado, medicamentos, supermercados, el problema de este último sector es que es más personalizado y a veces el sistema de pedidos puede fallar a los requerimientos del cliente.

Es conveniente destacar que la tendencia es que el mercado de entregas mediante aplicaciones móviles siga en crecimiento por lo menos para los próximos dos años para después entrar a un periodo de madurez, porque la entrada de nuevos clientes usando las Apps de pedido a casa está creciendo rápidamente y pronto una gran cantidad de personas que usan sus smartphones

van a tener y usar las aplicaciones de pedidos a casa.

\section{Conclusiones}

- Después de llevar a cabo el proceso de investigación y de haber realizado el análisis de los resultados obtenidos, para concluir, se procede a presentar las conclusiones del estudio.

- Las personas que más usan la aplicación móvil viene dado por aquellas que utilizan una única aplicación, así mismo la información recogida permitió determinar que la plataforma que más se usa en la ciudad de Cuenca, provincia 
del Azuay es la Rappi y Tipti, que representan el 37,30\%, se determinó que la mayor frecuencia en el uso de este servicio es frecuentemente por la noche para solicitar alimentos y medicamentos, por otra parte lo constituyen las tiendas y supermercado a domicilio, de allí que la plataforma digital que se utiliza permite ordenar desde un aparato móvil el listado de compras de los víveres y artículos para el hogar. Tipti es una aplicación 100\% ecuatoriana de compras de supermercado con entrega a domicilio, que trabaja con Tipti Shoppers. Los usuarios pueden descargarse la aplicación en App Store, Google Play, App Gallery. Los horarios de atención son sábados y domingos de 08:00 a 16:00.

- Es evidente que los hábitos de los consumidores en la ciudad de Cuenca, se han modificado de una manera drástica en cuanto al consumo de alimentos, víveres, medicación, se refiere, debido a la pandemia causada por COVID-19, no sólo porque entre los hábitos está el comprar menos productos de manera presencial, sino que el consumo de alimentos caseros se ha incrementado considerablemente, puesto que, el $73,40 \%$ se ha visto en esta necesidad tanto por la situación económica como sanitaria que se vive la ciudad, el país y el mundo.

- Los resultados evidencian que el comprar menos en tiendas aglomeradas es una prioridad para la gente actualmente, puesto que el $91,49 \%$ que adquieren alimentos, víveres, medicación, licores, artículos y herramientas y regalos, afirma la disminución de su consumo en este tipo de lugares, lo que representa una oportunidad tanto para estas empresas como para las aplicaciones de entrega de alimentos siempre y cuando ofrezcan promociones y descuentos que el usuario perciba como atractivas, de manera tal que permita la frecuencia de uso de las aplicaciones de entregas a domicilio.

- Con respecto a la tecnología que permite la comunicación entre dos aparatos que no están conectados por cables y que se basa en la transmisión y recepción de mensajes o señales por medio de ondas electromagnéticas, debemos confluir que las aplicaciones de entrega han aprovechado la tecnología para crear plataformas con total visibilidad y trazabilidad de una entrega de punto a punto, ya no necesitan cuestionar su fiabilidad. Las empresas han procurado reflejar las necesidades y preferencias de los consumidores locales en todas sus plataformas, ofreciendo el pago en efectivo o mediante la utilización de tarjetas de débito y/o crédito.

- Es de hacer notar que el servicio de delivery se distingue de los servicios típicos de compra convencional, como por ejemplo ir a las tiendas de mercado, farmacia, licorera, entre otros, porque integra una propuesta de valor para los usuarios que podrán realizar sus compras de una manera oportuna, práctica, fácil y diligente; con la cual se busca satisfacer las necesidades de los usuarios.

- La utilización de los medios digitales, incluyen computadora, tablet o móvil, software, imágenes digitales, video digital, videojuegos que constituyen medios 
codificados en un formato legible para una máquina, los mismos nos permiten crear, visualizar, distribuir, modificar y preservar en dispositivos electrónicos digitales, de allí que para obtener cualquier bien o servicio se requiere de tan solo un clic en estos tiempos de pandemia para adquirir un bien o servicio, sin la necesidad de salir de casa.

- Los emprendedores deben contar con recursos económicos para el inicio de su negocio, luego de ello, el marketing es muy importante, el acceso a las redes sociales es infinito, miles de millones de personas hacen compras online. Así mismo se puede decir que el capital humano es importante a la hora de emprender, personas con capacidad de visión de futuro, con compromiso y responsabilidad social, son importantes a la hora de determinar el éxito de un nuevo negocio.

- De la investigación se determinó, que el tiempo de entrega de los pedidos es quizás el driver, constituyéndose en uno de los componentes del software, que trabaja en conjunto con el sistema operativo y el controlador periférico, para proporcionar una interfaz funcional, permitiendo una propuesta de valor al cliente, que muy bien podría ser un factor decisivo de elección entre la utilización de una u otra compañía de servicio de delivery por aplicación dado que esto es lo que buscan al realizar solicitudes mediante estas plataformas buscando ahorrar tiempo y brinda la oportunidad de sumar un nuevo canal de ventas.

- Es innegable el efecto negativo que ha tenido la enfermedad pandémica en todo el mundo y el Ecuador no fue la distinción, la crisis sanitaria ha llevado que los inconvenientes económicos y sociales que ya se presentaban anterior a la enfermedad pandémica se profundicen todavía más, púes las medidas de confinamiento y distanciamiento han producido una recesión fundamental en las distintas ocupaciones económicas, es de esta forma que, a mayo 2020 se observa una caída significativa de las ventas, lo que se ve reflejado en los niveles de desempleo, puesto que las ocupaciones con niveles elevados y críticos de afectación, reducción en la recaudación de tributos son esas que contribuyen de forma fundamental tanto en el Producto Interno Bruto, como en el trabajo.

\section{Referencias Bibliográficas}

Arias, F. (2016). El Proyecto de Investigación: Introducción a la metodología científica. (7 $7^{\text {a }}$ Edición), Caracas - Venezuela. Editorial El pasillo, C.A.

Aldana, J. (2018). Big data: seguridad y gobernanza. Madrid: García-Maroto, Editores, SDB 004.6 ALD.

Becerra, E. \& Calle, O. (2020). El comercio electrónico en la región del Austro, como dinámica de la productividad. Conciencia Digital, 3(4), 6-25. 
Becerra, E., Cuadrado, G. \& Flores, M. (2021a). Medidas financieras en el entorno de los negocios, para minimizar los efectos de la pandemia COVID-19. Conciencia Digital, 4(3), 211-231.

Becerra, E., Jaramillo, Y., \& Astudillo, P. (2021b). La importancia de la información contable ante la incertidumbre del Covid-19, en las MiPymes en el entorno de los negocios en la región 6. Visionario Digital, 5(3), 111-132.

Becerra, E., Flores, M., \& Cuadrado, G. (2021c). La fiscalidad en las MiPymes de la región del Austro, en el contexto de la pandemia del COVID-19. Alpha publicaciones, 3(3), 98-119.

Becerra, E., Astudillo, P., \& Jaramillo, Y. (2021d) Alcance de la información societaria, durante la pandemia del COVID-19. Alfa Publicaciones, 3(3), 120-138.

El Observatorio Sectorial de la Restauración de DBK (2020), El servicio delivery: nuevos momentos de consumo, nuevas fuentes de ingreso, https://www.avanzafood.com/blog/el-servicio-delivery-nuevos-momentos-deconsumo-nuevas-fuentes-de-ingreso/

Euromonitor International (2020), El rotundo cambio del consumo de delivery por la pandemia del coronavirus, https://www.24horas.cl/economia/el-rotundocambio-del-consumo-de-delivery-por-la-pandemia-del-coronavirus-4178655

Hernández, S., Fernández, C., \& Baptista, L. (2016). Metodología de la Investigación. México: Mc Graw Hill Educación, 6ta. Edición.

Hurtado de Barrera, J. (2010). Guía para la comprensión holística de la ciencia, Tercera Edición, Fundación Sypal: Caracas-Venezuela

La Cámara Ecuatoriana de Comercio Electrónico- CECE. (2014). El comercio electrónico mantendrá un crecimiento sostenido en Ecuador, https://www.ekosnegocios.com/articulo/en-2021-el-comercio-electronicomantendra-un-crecimiento-sostenido-en-ecuador

La Cámara Ecuatoriana de Comercio Electrónico [CECE]. (2021). En el 2021, el comercio electrónico mantendrá un crecimiento sostenido en el país, https://revistagestion.ec/empresas/en-el-2021-el-comercio-electronicomantendra-un-crecimiento-sostenido-en-el-pais

La Comunidad Andina [CAN]. (2021). El 95\% del comercio de la Comunidad Andina de Naciones se enfoca en las manufacturas, https://www.larepublica.co/economia/el-95-del-comercio-de-la-comunidadandina-de-naciones-se-enfoca-en-las-manufacturas-2005817 


\section{VPDigital}

La Comisión Económica para América Latina y el Caribe [CEPAL]. (2019). El comercio digital en América Latina ¿Qué desafíos enfrentan las empresas y cómo superarlos, https://www.cepal.org/es/publicaciones/44976-comerciodigital-america-latina-que-desafios-enfrentan-empresas-como-superarlos

Ley de Comercio Electrónico, Firmas y Mensajes de Datos. (2014). Ley publicada en el Suplemento del Registro Oficial No 557, del 10 de febrero del 2014.

Ley Orgánica de Economía Popular y Solidaria (2018), Ley publicada en el Suplemento del Registro Oficial No 444, del 23 de octubre del 2018.

Reyes, F., Gómez, J., Vázquez, R., \& Chávez, H. (2021). Servicio de Entrega:

Tendencia de Hábitos y Consumo de Comida Mediante el uso de Aplicaciones Móviles. European Scientific Institute- ESJ, 17(4), 96-111.

Superintendencia de Compañías (2021), Ranking empresarial, https://appscvs.supercias.gob.ec/rankingCias/

Tamayo, M. (2003). El Proceso de Investigación Científica (4ª edición). México. Editorial Limusa.

\section{Ciencia}




\section{VP罟isiganital}

El artículo que se publica es de exclusiva responsabilidad de los autores y no necesariamente reflejan el pensamiento de la Revista Visionario Digital.

\section{¿Ciencia}

El artículo queda en propiedad de la revista y, por tanto, su publicación parcial y/o total en otro medio tiene que ser autorizado por el director de la Revista Visionario Digital.
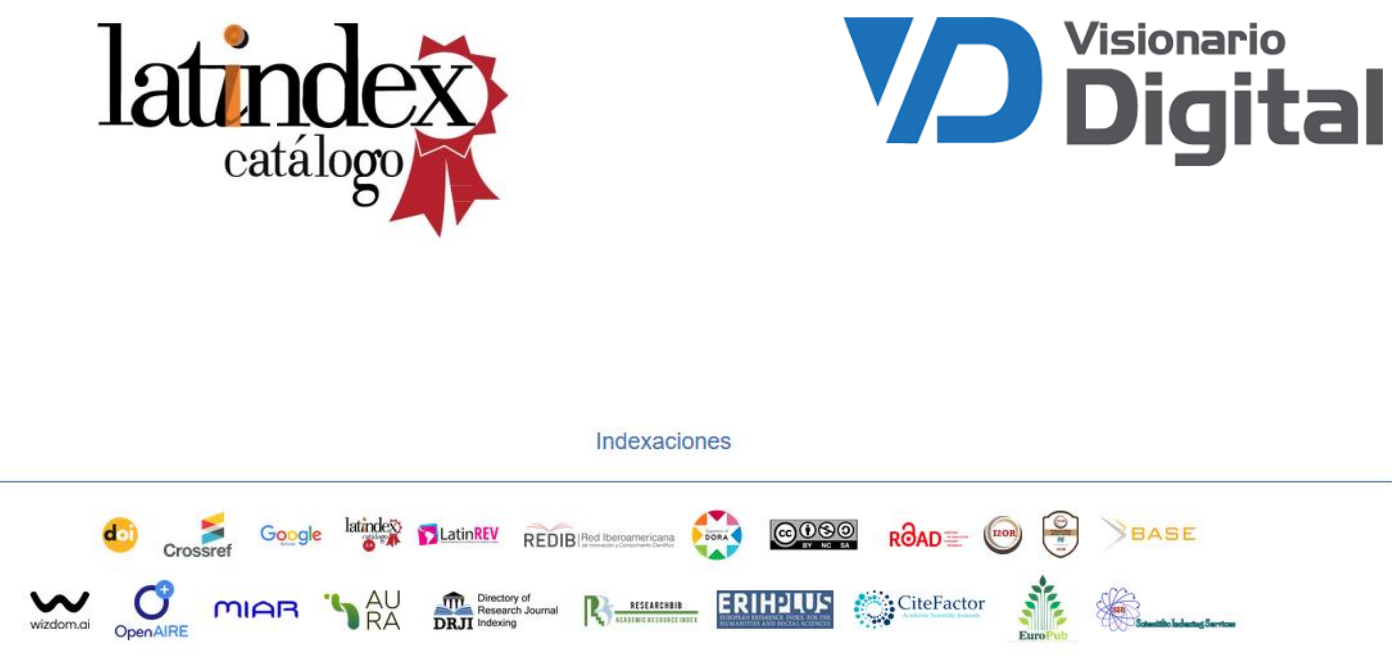